\title{
Seletividade de inseticidas utilizados em cultura cafeeira para larvas de Cryptolaemus montrouzieri Mulsant
}

\author{
Selectivity of insecticides used in the coffee crop to larvae of Cryptolaemus montrouzieri Mulsant
}

\author{
Luiz Carlos Dias Rocha' ${ }^{I}$ Geraldo Andrade Carvalho ${ }^{\text {II }}$ Valéria Fonseca Moscardini ${ }^{\text {II }}$ \\ Denise Tourino Rezende ${ }^{\text {III }}$
}

\section{RESUMO}

Assim como a maioria dos cultivos, em cafeeiro, a associação de inimigos naturais com produtos fitossanitários seletivos é uma importante estratégia no manejo integrado de pragas (MIP). Dessa forma, este estudo objetivou avaliar a seletividade de agrotóxicos utilizados na cultura cafeeira sobre larvas de Cryptolaemus montrouzieri Mulsant, 1853 (Coleoptera: Coccinellidae), em laboratório. Os bioensaios foram realizados em condições controladas $\left(25 \pm 2^{\circ} \mathrm{C}\right.$, UR de $70 \pm 10 \%$ e fotofase de 12 horas). Cada bioensaio consistiu em seis tratamentos e dez repetições, sendo cada parcela composta por duas larvas. Para as comparações das médias dos tratamentos, empregou-se um esquema fatorial inteiramente ao acaso de produtos $x$ número de instares para os diferentes instares subsequentes à aplicação. Os inseticidas utilizados $e$ suas respectivas dosagens de aplicação, em $\mathrm{g}$ de i.a. $\mathrm{L}^{-1}$ foram: tiametoxam $(0,5)$, imidacloprido $(0,7)$, óleo mineral $(13,3)$, endossulfam $(2,63)$ e dimetoato $(0,48)$. A testemunha foi composta apenas por água destilada. A aplicação dos produtos foi realizada por meio de torre de Potter. Avaliaram-se a sobrevivência dos espécimes após serem contaminados com os produtos e os efeitos dos compostos sobre os parâmetros reprodutivos do predador. Tiametoxam $(0,5)$, imidacloprido $(0,7)$ e endossulfam $(2,63)$ foram os mais prejudiciais, tendo sido observados, para os dois primeiros, $100 \%$ de mortalidade de larvas de primeiro e de segundo instares um dia após a aplicação e significativa redução na sobrevivência das larvas de terceiro e quarto instares. Dimetoato $(0,48)$ foi nocivo para larvas de primeiro instar e levemente nocivo para larvas de quarto instar. Em função da seletividade apresentada pelo óleo mineral $(13,3)$, este pode ser recomendado em compatibilização com o predador C. montrouzieri em programas de MIP na cultura cafeeira, sem prejuízo ao desempenho do inimigo natural
Palavras-chave: insetos sugadores, joaninha, agrotóxicos, café, seletividade, controle biológico.

\section{ABSTRACT}

As well as most of the crops, in coffee crop, the association of selective compounds and natural enemies, composes an important tool for the integrated pest management (IPM). The objective of this study was to evaluate the selectivity of some pesticides used in coffee crops on larvae of Cryptolaemus montrouzieri Mulsant, 1853 (Coleoptera: Coccinellidae) under controlled laboratory conditions (climatic chamber) at $25 \pm 2^{\circ} \mathrm{C}, \mathrm{RH}$ of $70 \pm 10 \%$ with a 12 h-photophase. The randomized experimental design was used, with six treatments and ten repetitions, being each composed for two larvae. For the comparisons of the averages of treatments, a factorial scheme was used with the products $x$ number of instars for the different instars subsequent the application. The pesticides and doses in $g$ of a.i. $L^{-1}$ were: thiamethoxan (0.5), imidacloprid (0.7), mineral oil (13.3), endosulfan (2.63) and dimethoate (0.48). Distilled water was used as control. The sprayings of the pesticides were accomplished using a Potter's tower. The parameters evaluated were: specimens survival after the application of the compounds, and the compounds effects over the reproductive parameters of the predator. Thiamethoxan (0.5), imidacloprid (0.7) and endosulfan (2.63) were the most harmful to the treated individuals and for the first two compounds it was observed $100 \%$ of larvae mortality of the first and second instars soon at 1 day after the application and reduction in the survival of the larvae of third and fourth instars. Dimethoate (0.48) was harmful for the larvae of the first instar and slightly harmful for larvae of fourth instar. As a function of the selectivity presented by mineral oil (13.3), it can be recommended aiming its

Instituto Federal do Sul de Minas (IFSULDEMINAS), Campus Inconfidentes, Praça Tiradentes, 416, 37576-000, Inconfidentes, MG, Brasil. E-mail: luiz.ifet@gmail.com. Autor para correspondência.

IIDepartamento de Entomologia, Universidade Federal de Lavras (UFLA), Lavras, MG, Brasil.

IIIDefesa Fitossanitária, Departamento de Produção Vegetal, Universidade Estadual Paulista (UNESP), Botucatu, SP, Brasil. 
compatibilization with the $\boldsymbol{C}$. montrouzieri natural enemy in programs of IPM in coffee crop.

Key words: sucking insects, ladybeetles, pesticides, coffee, selectivity, biological control.

\section{INTRODUÇÃO}

No Brasil, a cultura cafeeira vem sendo atacada por diversas espécies de cochonilhas. As cochonilhas-brancas Planococcus citri (Risso, 1813) (Hemiptera: Pseudococcidae) e Planococcus minor (Maskell, 1897) (Hemiptera: Pseudococcidae) e a cochonilha-branca-de-cauda-longa Pseudococcus longispinus (Targioni Tozzetti, 1867) (Hemiptera: Pseudococcidae) são as cochonilhas-farinhentas mais comuns na parte aérea dos cafeeiros. Suas ninfas ou fêmeas adultas sugam a seiva nas rosetas, levando ao chochamento e à queda de botões florais e frutos ainda em desenvolvimento (SANTA-CECÍLIA et al., 2007).

O controle dessas pragas vem sendo realizado de formas variadas e, nos últimos anos, táticas de controle preconizadas no manejo integrado de pragas (MIP) têm sido cada vez mais empregadas nessa cultura, principalmente em sistemas de produção integrada de café (PIC), que se encontra em fase de implantação no território brasileiro (BOLLER et al., 1999; ANDRIGUETO \& KOSOSKI, 2002; RAIJ, 2003; BOLLER et al., 2004). Nesse sistema, preconiza-se, entre outras práticas de proteção ao meio ambiente, a redução no uso de produtos fitossanitários e a intensificação do emprego de outros métodos para o controle de pragas, visando à obtenção de produtos de melhor qualidade. Dessa forma, o uso de inimigos naturais, como predadores e ou parasitoides, está sendo bastante incentivado.

Em diversos países da região Neotropical, a joaninha-superpredadora, como é conhecida a Cryptolaemus montrouzieri Mulsant, 1853 (Coleoptera: Coccinellidae), é utilizada no controle de hemípteros, principalmente para o controle de cochonilhas (Hemiptera: Pseudococcidae) (NARDO et al., 1999). Apesar dessa importância do controle biológico, o controle químico ainda é necessário em muitas situações. Entretanto, ainda são poucos os trabalhos relacionados ao efeito de produtos fitossanitários sobre $\boldsymbol{C}$. montrouzieri, embora, até 1990 , tenha sido considerado o $15^{\circ}$ inimigo natural mais estudado quanto à seletividade (CROFT, 1990). Dessa maneira, considerando-se a potencialidade dos predadores da espécie $\boldsymbol{C}$. montrouzieri no controle de cochonilhas em cafeeiros e a necessidade da compatibilização desses organismos com aplicações de agrotóxicos, o objetivo da realização deste trabalho foi avaliar a seletividade fisiológica de inseticidas utilizados na cultura cafeeira para esse predador.

\section{MATERIAL E MÉTODOS}

\section{Realização dos bioensaios}

Os bioensaios seguiram a metodologia proposta por membros da International Organization for Biological Control (IOBC) para uso com as espécies Aleochara bilineata (Gyllenhal, 1810) (Coleoptera: Staphylinidae), Coccinella septempunctata (Linnaeus, 1758) (Coleoptera: Coccinellidae), Adalia bipunctata (Linnaeus, 1758) (Coleoptera: Coccinellidae), Propylea quatuordecimpunctata (Linnaeus, 1758) (Coleoptera: Coccinellidae) e Harmonia axyridis (Pallas, 1773) (Coleoptera: Coccinellidae) (SAMSØE-PETERSEN, 1992; JANSEN \& HAUTIER, 2006), porém, neste estudo, adaptada para a espécie $\boldsymbol{C}$. montrouzieri. Os compostos utilizados e suas respectivas dosagens de aplicação, em g de i.a. $L^{-1}$ foram: tiametoxam $(0,5)$, imidacloprido $(0,7)$, óleo mineral $(13,3)$, endossulfam $(2,63)$ e dimetoato $(0,48)$. A testemunha foi constituída apenas por água destilada.

Os espécimes empregados nos bioensaios (ovos e larvas) foram provenientes da criação de laboratório. Os insetos foram individualizadas em placas de Petri de $5 \mathrm{~cm}$ de diâmetro, vedadas com filme de PVC transparente. As larvas foram alimentadas à vontade a cada dois dias, com $\boldsymbol{P}$. citri e mantidas em câmara climatizada regulada a $25 \pm 2^{\circ} \mathrm{C}$, UR de $70 \pm 10 \%$ e 12 horas de fotofase. Após cerca de 24 horas da eclosão ou da mudança de instar, vinte larvas de primeiro, segundo, terceiro ou quarto instares para cada tratamento, foram separadas em placa de Petri de $15 \mathrm{~cm}$ de diâmetro e receberam os inseticidas via pulverização em torre de Potter. As pulverizações dos compostos foram realizadas diretamente sobre os espécimes por meio de torre de Potter, com a aplicação de $1,5 \pm 0,5 \mathrm{mg} \mathrm{cm}^{-2}$.

A sobrevivência das larvas foi avaliada logo após aplicação (1 hora) e 1, 2, 3, 4 e 5 dias após aplicação dos produtos, com o auxílio de um microscópio estereoscópico (40x). Foi considerada morta a larva que se manteve imóvel ao estímulo gerado pelo toque de um pincel. A avaliação da oviposição foi realizada para fêmeas adultas oriundas de larvas tratadas em todos os instares. Os adultos foram separados em casais e acondicionados em placas de Petri de $5 \mathrm{~cm}$ de diâmetro e fechadas com filme de PVC transparente. Os espécimes foram alimentados à vontade por meio do fornecimento de água (em algodão umedecido) e ovos, ninfas e adultos da cochonilha $\boldsymbol{P}$. citri. Para estudos da viabilidade dos ovos, foram coletados, ao acaso, 30 ovos de cada tratamento a cada semana, por um período de 20 dias. 
Análises estatísticas

O delineamento foi o inteiramente ao acaso e utilizou-se um esquema fatorial de produtos x número de instares com dez repetições, utilizando-se duas larvas em cada unidade experimental, sendo: fatorial de $6 \times 4$, para larvas tratadas no primeiro instar e fase de ovo; fatorial de 6x3, para larvas de segundo instar e; fatorial de 6x2, para larvas de terceiro. Avaliaram-se a sobrevivência das larvas logo após aplicação (1 hora) e 1, 2, 3, 4 e 5 dias após a aplicação e parâmetros reprodutivos de adultos oriundos dos insetos tratados.

Os dados de sobrevivência logo após aplicação dos compostos e do $1^{\circ}$ ao $5^{\circ}$ dia após a aplicação sobre as larvas nos diferentes instares foram, em função da alta variabilidade, transformados para arcoseno $(\sqrt{ } \mathrm{x} / 100)$ e submetidos à análise de variância em um modelo de parcelas subdivididas no tempo, com os produtos na parcela. As comparações das médias dos tratamentos foram realizadas por meio do teste de Scott e Knott a 5\% de significância (SCOTT \& KNOTT, 1974).

Para os dados balanceados, foi utilizado o programa estatístico Sisvar (FERREIRA, 2000). Quando o número de repetições foi diferente para os tratamentos, utilizou-se o proc GLM do SAS (SAS INSTITUTE, 2001).

Classificação dos produtos quanto à toxicidade segundo escala da IOBC

Os produtos foram enquadrados em classes de toxicidade conforme as recomendações sugeridas por membros da IOBC (HASSAN, 1992; SAMSØEPETERSEN, 1992; HASSAN \& DEGRANDE, 1996; VEIRE et al., 2002; JANSEN \& HAUTIER, 2006), em que classe 1 = inócuo $(\mathrm{E}<30 \%)$, classe 2 = levemente nocivo ( $30 \% \leq \mathrm{E} \leq 80 \%$ ), classe $3=$ moderadamente nocivo $(80 \%<\mathrm{E} 99 \%)$ e classe $4=$ nocivo $(\mathrm{E}>99 \%)$, em função do efeito sobre os parâmetros reprodutivos e mortalidade do predador, sendo o efeito total (E\%) calculado pela fórmula proposta por VOGT (1992): $E=100-(100-M) \times R_{1} \times R_{2}$

Em que: $\mathrm{E}=$ efeito total (\%); $\mathrm{M} \%=$ mortalidade total, corrigida em função do tratamento testemunha (ABBOTT, 1925); $\mathrm{R}_{1}=$ razão entre a média diária de ovos colocados por fêmea tratada e não tratada; $\mathrm{R}_{2}=$ razão entre a média de ovos viáveis colocados por fêmea tratada e não tratada.

\section{RESULTADOS E DISCUSSÃO}

Efeito dos inseticidas na sobrevivência de larvas de $\boldsymbol{C}$. montrouzieri

Os efeitos de tiametoxam $(0,5)$ e imidacloprido $(0,7)$ foram perceptíveis logo na primeira hora após a pulverização, com redução na sobrevivência das larvas, não sendo observada a presença de nenhum inseto tratado sobrevivente na avaliação de 1 dia. Óleo mineral $(13,3)$, endossulfam $(2,63)$ e dimetoato $(0,48)$, um dia após pulverização, promoveram mortalidade significativa dos insetos tratados; para endossulfam $(2,63)$, observou-se que o efeito ocorreu também ao longo do período de avaliações, com sobrevivência média de 13,3\%, cinco dias após a sua aplicação (Tabela 1).

De acordo com TOMIZAWA \& CASIDA (2003) e TOMIZAWA \& CASIDA(2005), os inseticidas neonicotinoides atuam como agonistas no receptor pós-sináptico nicotínico da acetilcolina (nAChR) em insetos. Nos insetos, o nAChR encontra-se amplamente distribuído e é predominante nas regiões neuropil do sistema nervoso central. Esse receptor não é apenas responsável por uma neurotransmissão rápida, mas é também um importante alvo para a ação desses inseticidas, o que explica a rápida ação e a elevada mortalidade causada por esses compostos no presente estudo. No entanto, os efeitos de alguns compostos, principalmente de inseticidas neonicotinoides sobre C. montrouzieri ainda são pouco conhecidos.

A pulverização dos produtos fitossanitários sobre larvas de segundo instar resultou em efeito tóxico de todos os produtos a partir do primeiro dia após aplicação; entretanto, para tiametoxam $(0,5)$ e imidacloprido $(0,7)$, o efeito foi evidenciado já logo após a pulverização (Tabela 1). A partir do segundo dia, observaram-se valores constantes de sobrevivência dos insetos, porém, o efeito prejudicial do endossulfam $(2,63)$ foi verificado até o final das avaliações, cinco dias após a pulverização. Endossulfam $(2,63)$ pertence ao grupo dos ciclodienos, cujas substâncias podem apresentar capacidade de bioacumulação (WARE \& WHITACRE, 2004) e apresentar seu efeito posteriormente, o que pode explicar os resultados obtidos.

Larvas de terceiro instar submetidas à pulverização dos inseticidas também tiveram a sobrevivência reduzida, assim como observado para os instares anteriores. Entretanto, para os insetos tratados com óleo mineral no terceiro instar, não se observou efeito tóxico. Tiametoxam $(0,5)$ foi o produto mais tóxico, provocando $100 \%$ de mortalidade das larvas de terceiro instar um dia após a pulverização. Imidacloprido $(0,7)$, endossulfam $(2,63)$ e dimetoato $(0,48)$ também reduziram a sobrevivência das larvas, apresentando médias de 30,8\%; 46,2\% e 38,5\%, respectivamente, ao final das avaliações desta característica biológica (Tabela 2).

Ao longo do período de avaliação, constatou-se que imidacloprido $(0,7)$ e dimetoato $(0,48)$ 
Tabela 1 - Sobrevivência (\%) ( \pm EP) de larvas de primeiro e segundo instares de Cryptolaemus montrouzieri tratadas com produtos fitossanitários. Temperatura de $25 \pm 2^{\circ} \mathrm{C}$, UR de $70 \pm 10 \%$ e fotofase de 12 horas.

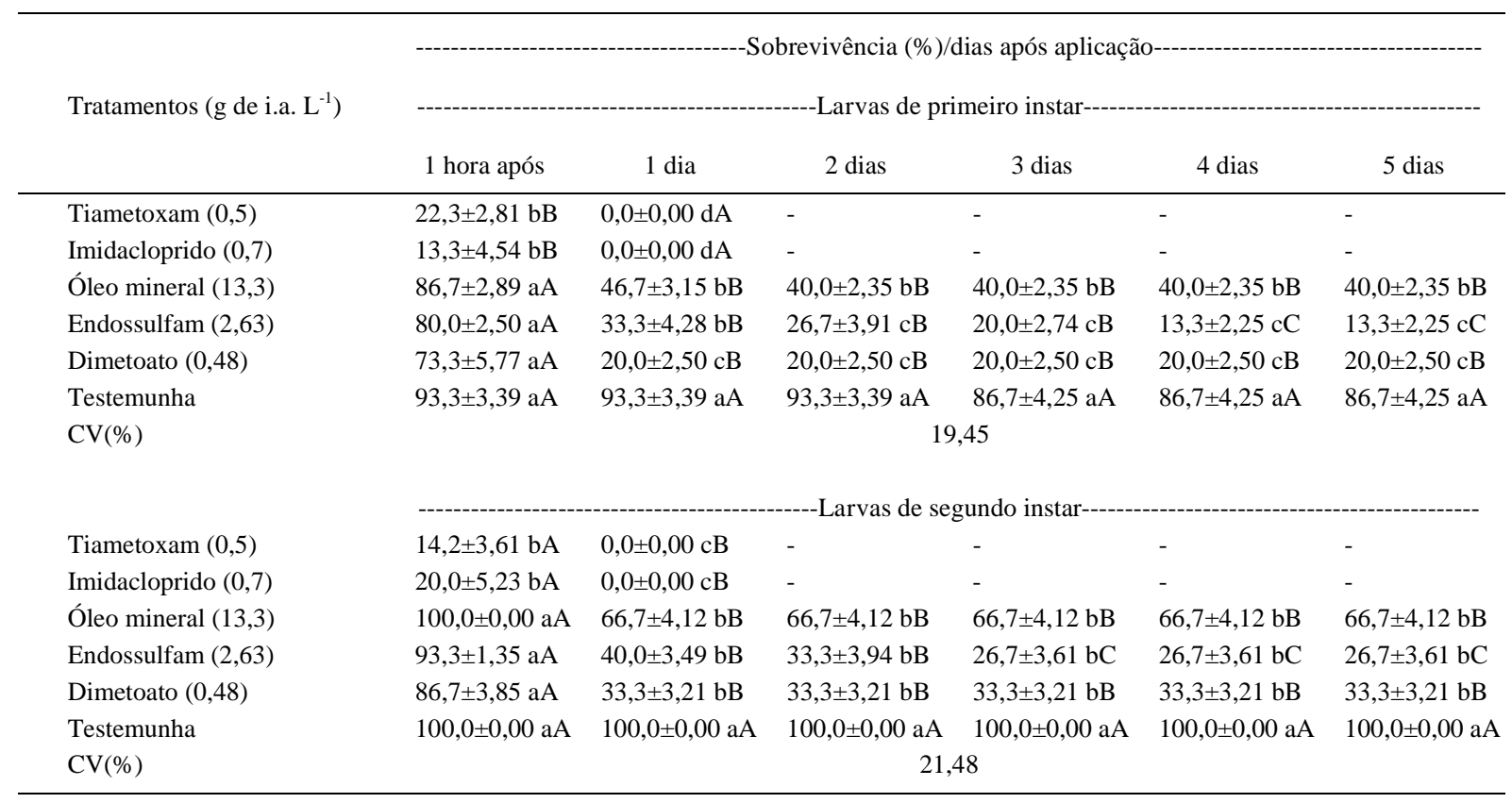

Médias seguidas pela mesma letra, minúsculas na coluna e maiúsculas na linha, não diferem estatisticamente entre si, pelo teste de Scott e Knott $(\mathrm{P}<0,05)$. (-) Número de insetos insuficientes para análise deste parâmetro.

provocaram mortalidade de espécimes de terceiro instar tratados e, diferente do que foi observado para larvas de primeiro e segundo instares, endossulfam $(2,63)$ provocou efeito prejudicial apenas no primeiro dia de avaliação e não se observou efeito ao longo das avaliações para larvas de terceiro estádio. A maior capacidade dos insetos de estádios mais adiantados de desenvolvimento em suportar o contato com os produtos fitossanitários foi relatada por CROFT (1990). O aumento de reservas, a capacidade de eliminação e ou a capacidade de degradação do produto podem estar relacionados à manifestação desta característica nos espécimes.

Uma hora após a pulverização, não foi verificado efeito tóxico de nenhum dos compostos sobre as larvas de quarto instar de $\boldsymbol{C}$. montrouzieri. No entanto, a partir da avaliação realizada um dia após a pulverização dos produtos, verificou-se efeito prejudicial de tiametoxam $(0,5)$, imidacloprido $(0,7)$ e endossulfam $(2,63)$, os quais reduziram a sobrevivência das larvas. Dimetoato $(0,48)$ somente apresentou toxicidade significativa 48 horas após a sua aplicação, com média de 73,3\% de sobrevivência (Tabela 2).

Em todas as avaliações realizadas, não se verificou efeito tóxico do óleo mineral $(13,3)$ sobre as larvas de quarto instar, revelando-se seletivo para esse estádio de desenvolvimento (Tabela 2). O óleo mineral é recomendado para o controle de diversas pragas agrícolas de importantes culturas, como cafeeiro, citros, macieira, pessegueiro, entre outras, sendo utilizado em aplicação foliar como inseticida, acaricida e também como fungicida, atuando inclusive como diluente, adjuvante e espalhante adesivo. Seu efeito sobre artrópodes-praga, por exemplo, pode estar associado à sua capacidade de penetrar através da cutícula desses organismos e, quando em associação com inseticidas, apresentam efeito sinergístico, potencializando a capacidade de penetração dos compostos tóxicos e aumentando a ação tóxica destes (FERNANDEZ, et al., 2005; MANZONI et al., 2006; MAPA, 2010). No entanto, em relação à sua ação isolada sobre larvas de primeiro e segundo instares de $\boldsymbol{C}$. montrouzieri, como observado no presente estudo, parece estar associada a um efeito mecânico, atuando diretamente nos espiráculos das larvas, causando mortalidade desses organismos por anoxia. Tal efeito não foi observado para larvas de terceiro e quarto instares dessa espécie, possivelmente por se tratarem de organismos de maior tamanho corporal.

Desde o início da segunda metade do século passado, trabalhos como o de RIPPER et al. (1951) têm buscado compreender o efeito dos agrotóxicos sobre os inimigos naturais. Entretanto, nos últimos anos, as pesquisas têm dado ênfase não só para a mortalidade 
Tabela 2 - Sobrevivência (\%) ( \pm EP) de larvas de terceiro e quarto instares de Cryptolaemus montrouzieri tratadas com produtos fitossanitários. Temperatura de $25 \pm 2^{\circ} \mathrm{C}$, UR de $70 \pm 10 \%$ e fotofase de 12 horas.

\begin{tabular}{|c|c|c|c|c|c|c|}
\hline \multirow[t]{2}{*}{ Tratamentos (g de i.a. $\mathrm{L}^{-1}$ ) } & \multicolumn{6}{|c|}{-------------------------------------------------Larvas de terceiro instar----------------------------------------------- } \\
\hline & 1 hora após & 1 dia & 2 dias & 3 dias & 4 dias & 5 dias \\
\hline Tiametoxam $(0,5)$ & $30,8 \pm 6,35$ bA & $0,0 \pm 0,00 \mathrm{cB}$ & - & - & - & - \\
\hline Imidacloprido $(0,7)$ & $61,5 \pm 4,27$ bA & $53,8 \pm 4,19$ bA & $53,8 \pm 4,19$ bA & $53,8 \pm 4,19$ bA & $46,2 \pm 3,89 \mathrm{bB}$ & $30,8 \pm 4,01$ bC \\
\hline Óleo mineral $(13,3)$ & $100,0 \pm 0,00 \mathrm{aA}$ & $100,0 \pm 0,00 \mathrm{aA}$ & $100,0 \pm 0,00 \mathrm{aA}$ & $100,0 \pm 0,00 \mathrm{aA}$ & $100,0 \pm 0,00 \mathrm{aA}$ & $100,0 \pm 0,00 \mathrm{aA}$ \\
\hline Endossulfam $(2,63)$ & $84,6 \pm 3,08 \mathrm{aA}$ & $53,8 \pm 4,03 \mathrm{bB}$ & $46,2 \pm 3,77 \mathrm{bB}$ & $46,2 \pm 3,77$ bB & $46,2 \pm 3,77 \mathrm{bB}$ & $46,2 \pm 3,77$ bB \\
\hline Dimetoato $(0,48)$ & $100,0 \pm 0,00 \mathrm{aA}$ & $84,6 \pm 3,22$ aA & $69,2 \pm 2,89 \mathrm{bB}$ & $46,2 \pm 2,45 \mathrm{bC}$ & $46,2 \pm 2,45 \mathrm{bC}$ & $38,5 \pm 3,36$ bC \\
\hline Testemunha & $100,0 \pm 0,00 \mathrm{aA}$ & $100,0 \pm 0,00 \mathrm{aA}$ & $100,0 \pm 0,00 \mathrm{aA}$ & $92,3 \pm 1,47$ aA & $92,3 \pm 1,47$ aA & $92,3 \pm 1,47$ aA \\
\hline $\mathrm{CV}(\%)$ & \multicolumn{6}{|c|}{16,52} \\
\hline Tiametoxam $(0,5)$ & $87,9 \pm 4,37$ aA & $20,0 \pm 4,97 \mathrm{cB}$ & $13,3 \pm 2,89 \mathrm{~dB}$ & $13,3 \pm 2,89 \mathrm{cB}$ & $13,3 \pm 2,89 \mathrm{cB}$ & $6,7 \pm 4,74 \mathrm{cB}$ \\
\hline Imidacloprido $(0,7)$ & $89,3 \pm 5,03 \mathrm{aA}$ & $53,3 \pm 3,55$ bB & $53,3 \pm 3,55 \mathrm{cB}$ & $53,3 \pm 3,55$ bB & $46,7 \pm 4,22$ bB & $40,0 \pm 3,90 \mathrm{bB}$ \\
\hline Óleo mineral $(13,3)$ & $100,0 \pm 0,00 \mathrm{aA}$ & $93,3 \pm 4,66$ aB & $86,7 \pm 3,33 \mathrm{aB}$ & $86,7 \pm 3,33 \mathrm{aB}$ & $86,7 \pm 3,33 \mathrm{aB}$ & $86,7 \pm 3,33 \mathrm{aB}$ \\
\hline Endossulfam $(2,63)$ & $86,7 \pm 2,33$ aA & $53,3 \pm 4,01 \mathrm{bB}$ & $53,3 \pm 4,01 \mathrm{cB}$ & $53,3 \pm 4,01 \mathrm{bB}$ & $53,3 \pm 4,01 \mathrm{bB}$ & $53,3 \pm 4,01$ bB \\
\hline Dimetoato $(0,48)$ & $100,0 \pm 0,00 \mathrm{aA}$ & $86,7 \pm 6,20 \mathrm{aB}$ & $73,3 \pm 5,22 \mathrm{bB}$ & $46,7 \pm 4,39$ bC & $46,7 \pm 4,39 \mathrm{bC}$ & $40,0 \pm 3,48 \mathrm{bC}$ \\
\hline Testemunha & $93,3 \pm 4,31 \mathrm{aA}$ & $93,3 \pm 4,31$ aA & $93,3 \pm 4,31 \mathrm{aA}$ & $86,7 \pm 3,59$ aA & $86,7 \pm 3,59$ aA & $86,7 \pm 3,59$ aA \\
\hline CV(\%) & \multicolumn{6}{|c|}{25,12} \\
\hline
\end{tabular}

Médias seguidas pela mesma letra, minúsculas na coluna e maiúsculas na linha, não diferem estatisticamente entre si, pelo teste de Scott e Knott $(\mathrm{P}<0,05)$. (-) Número de insetos insuficientes para análise deste parâmetro.

de espécimes adultos, mas também às diferentes fases de desenvolvimento e, ainda, aos efeitos subletais que os compostos podem provocar nos organismos nãoalvos, como apresentado no presente estudo.

Os resultados aqui obtidos, para larvas, permitiram a separação dos compostos tiametoxam $(0,5)$ e imidacloprido $(0,7)$, os quais foram nocivos (classe 4 ) para quase todos os estádios de $\boldsymbol{C}$. montrouzieri avaliados. Tiametoxam $(0,5)$ e imidacloprido $(0,7)$ são compostos pertencentes ao grupo dos neonicotinoides e atuam por contato e ingestão, apresentando também ação translaminar e atividade sistêmica em tecidos de plantas (PMRA, 2001). Os compostos neonicotinoides são potentes agonistas da acetilcolina nas junções colinérgicas do sistema nervoso dos insetos e, como não são degradados pela acetilcolinesterase, provocam a morte dos insetos (WARE \& WHITACRE, 2004). Esta ação foi observada em larvas de $\boldsymbol{C}$. montrouzieri de forma severa, quando expostas aos produtos tiametoxam $(0,5)$ e imidacloprido $(0,7)$.

Efeito total dos produtos fitossanitários sobre o predador de acordo com escala da IOBC

A alta mortalidade apresentada pelos compostos neonicotinoides, com exceção do imidacloprido $(0,7)$ para larvas de quarto estádio, resultou na classificação desses compostos como nocivos ao predador (classe 4), quando aplicados sobre larvas (Tabela 3). Ainda nessa tabela, é possível observar que ocorreu maior sensibilidade dos espécimes tratados no primeiro e segundo instares. A mortalidade provocada por tiametoxam $(0,5)$, imidacloprido $(0,7)$, endossulfam $(2,63)$ e dimetoato $(0,48)$ foi elevada, sendo considerados nocivos (classe 4). Destes, apenas o último permitiu a avaliação dos parâmetros reprodutivos de fêmeas provenientes de larvas tratadas no segundo instar e foi enquadrado na classe 3.

As larvas de terceiro e de quarto instares foram mais tolerantes ao endossulfam $(2,63)$ e dimetoato $(0,48)$, os quais foram considerados levemente nocivos, com valores de efeito total da ordem de 66,5\% e 81,8\% e de $75,0 \%$ e $75,9 \%$, respectivamente (Tabela 3 ). Novamente, não se observou efeito prejudicial do óleo mineral ao predador, sendo pertencente à classe 1 .

Dentre os compostos neurotóxicos, os organofosforados apresentam elevado impacto sobre os coccinelídeos. Em dezessete compostos deste grupo já testados, a média de toxicidade calculada variou de 2,8 a 3,0 na escala proposta pela IOBC, que varia de 1 até 4 (MIRET \& GARCÍA-MARÍ, 2001). Em ensaios realizados por BOYERO et al. (2005), os compostos organofosforados metidatiom e malatiom (em concentrações de $0,15 \%$ e $0,6 \%$, respectivamente) causaram elevada mortalidade de $\boldsymbol{C}$. montrouzieri, 24 
Tabela 3 - Mortalidade (\%), oviposição, efeito total (E\%) e classificação dos produtos, em função da escala de toxicidade proposta pela IOBC, de espécimes oriundos de Cryptolaemus montrouzieri tratados no primeiro, segundo, terceiro e quarto instares.

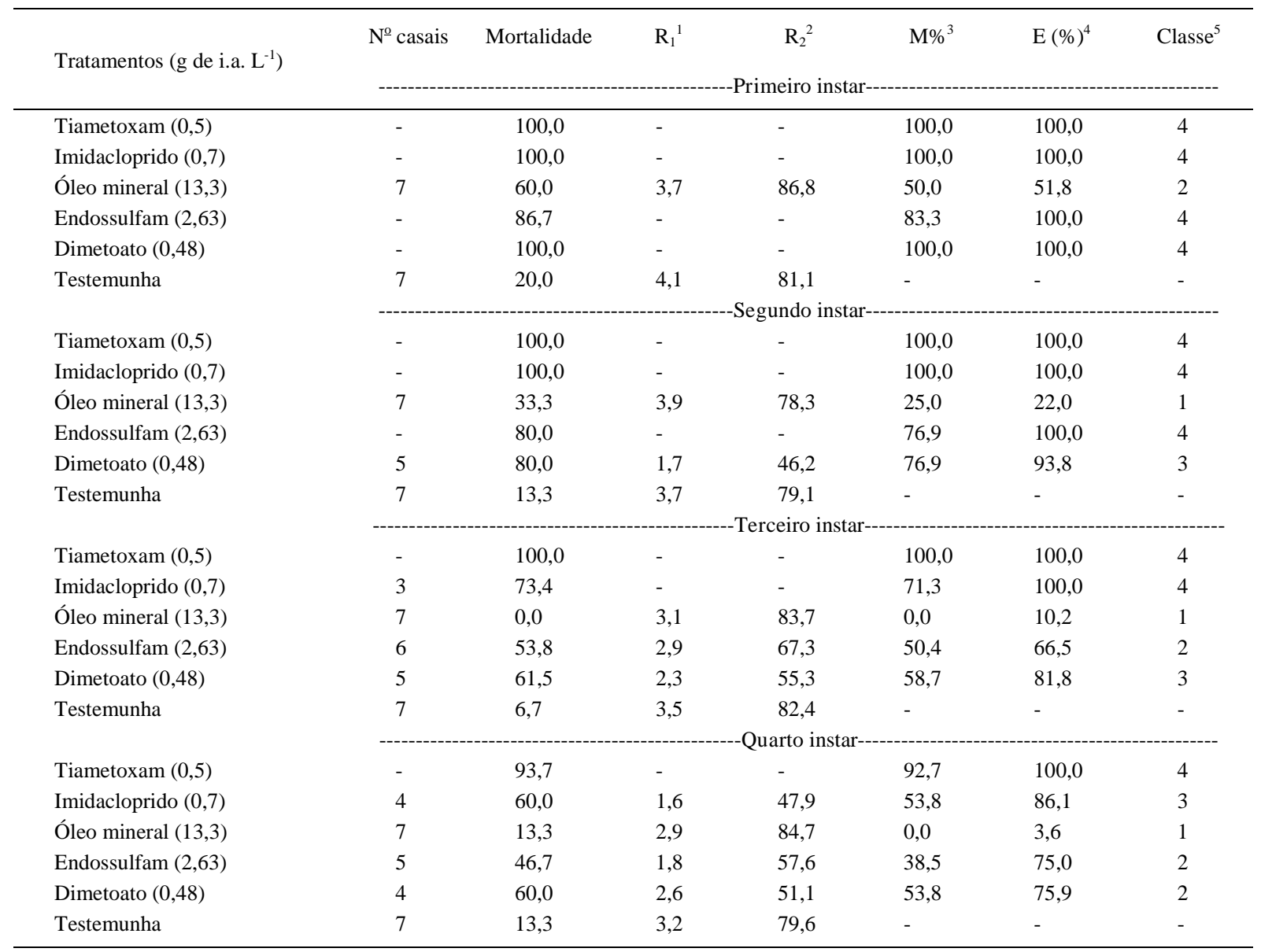

${ }^{1} \mathrm{R}_{1}$ : Oviposição média/fêmea/dia; ${ }^{2} \mathrm{R}_{2}$ : Viabilidade de ovos (\%); ${ }^{3} \mathrm{M} \%$ : Mortalidade no tratamento corrigida pela fórmula de ABBOTT (1925); ${ }^{4} \mathrm{E} \%$ : Efeito total do produto sobre o predador. $\mathrm{E}=100 \%-(100 \%-\mathrm{M} \%) \times \mathrm{R}_{1} \times \mathrm{R}_{2}$.

${ }^{5}$ Classe de toxicidade: classe 1 = inócuo ( $\mathrm{E}<30 \%$ ), classe 2 = levemente nocivo (30\% $\left.\leq \mathrm{E} \leq 80 \%\right)$, classe $3=$ moderadamente nocivo $(80 \%<E \leq 99 \%)$ e classe 4 = nocivo (E>99\%) (VEIRE et al., 2002). (-) Número de insetos insuficientes para análise deste parâmetro.

horas após a sua aplicação. Esses resultados assemelham-se aos obtidos neste estudo para dimetoato, em que 24 horas após a sua pulverização, a mortalidade dessa espécie de predador foi de cerca de $70 \%$. De acordo com BELLOWS et al. (1985), dimetoato apresentou degradação inicial rápida, com meia vida de 2,2 dias, quando aplicado em superfície foliar de Citrus limom (Linnaeus), porém, ainda assim, o contato cinco dias após a sua aplicação causou mortalidade de C. montrouzieri superior a 50\%.

Entre os produtos testados, óleo mineral $(13,3)$ revelou-se pouco tóxico, assemelhando-se aos resultados alcançados por MORSE et al. (1987), os quais submeteram adultos de $\boldsymbol{C}$. montrouzieri a resíduos desse produto e não observaram diferenças significativas em relação ao tratamento controle. Assemelharam-se também aos de DIB (1998), em que foi classificado como levemente nocivo (classe 2). Com relação ao imidacloprido $(0,7)$, este autor constatou efeito prejudicial sobre $\boldsymbol{C}$. montrouzieri, sendo classificado como medianamente nocivo e, além disso, verificou-se que o composto foi ligeiramente persistente, apresentando toxicidade significativa por um período de 11 a 14 dias.

A ação direta e indireta de neonicotinoides sobre $\boldsymbol{C}$. montrouzieri foi estudada por CLOYD \& DICKINSON (2006), cujos resultados evidenciaram mortalidade elevada 24 horas após a aplicação dos compostos. Decorridas 48 horas, a mortalidade atingiu $100 \%$ para os insetos dos tratamentos à base de acetamiprido e clotianidino (enquadrados na classe 4). Esses agrotóxicos pertencem ao mesmo grupo químico de imidacloprido $(0,7)$ e tiametoxam $(0,5)$, avaliados neste estudo, com resultados bastante semelhantes. 
Também para imidacloprido $(0,7)$ e tiametoxam $(0,5)$, aplicados sobre ovos e adultos de $\boldsymbol{C}$. montrouzieri, ROCHA et al. (2010) constataram elevada mortalidade, sendo enquadrados na classe 4, como nocivos.

Na literatura, são escassos os estudos que abordam os efeitos de tiametoxam $(0,5)$ sobre esse predador e, em função do seu efeito prejudicial constatado no presente trabalho, novas pesquisas deverão ser realizadas para confirmar ou não a sua toxicidade para este inimigo natural, em condições de semicampo e campo. É importante ressaltar, ainda, que este composto é recomendado para aplicação via solo na cultura cafeeira, podendo, esta forma de aplicação, conferir seletividade ecológica para alguns inimigos naturais presentes no agroecossistema cafeeiro.

\section{CONCLUSÃO}

Endossulfam $(2,63)$ é nocivo para $C$. montrouzieri, quando aplicado sobre larvas de primeiro e de segundo instares, e é levemente nocivo para larvas de terceiro e quarto instares. Tiametoxam $(0,5)$ e imidacloprido $(0,7)$ são nocivos para larvas de $\boldsymbol{C}$. montrouzieri em todos os estádios de desenvolvimento. Para larvas de quarto instar, imidacloprido (0,7) é moderadamente nocivo. Dimetoato $(0,48)$ é nocivo para larvas de primeiro instar, levemente nocivo para larvas de quarto instar e moderadamente nocivo para larvas de segundo e terceiro instares de $\boldsymbol{C}$. montrouzieri.

Óleo mineral $(13,3)$ é pouco prejudicial para C. montrouzieri e, portanto, pode ser empregado em programas de manejo integrado de cochonilhas da parte aérea de cafeeiros, visando à preservação desse predador.

\section{REFERÊNCIAS}

ABBOTT, W.S. A method of computing the effectiveness of an insecticide. Journal of Economic Entomology, Lanham, v.18, n.2, p.265-267, 1925.

ANDRIGUETO, J.R.; KOSOSKI, A.R. Marco legal da produção integrada de frutas no Brasil. Brasília: MAPA/SARC, 2002. 60p. Disponível em: <http://www.cnpuv.embrapa.br/tecnologias/ pim/marco_legal.pdf $>$. Acesso em: $11 \mathrm{dez} .2008$.

BELLOWS, T.S.J. et al. Residual toxicity of four insecticides used for control of citrus thrips (Thysanoptera: Thripidae) on three beneficial species in a citrus agroecosystem. Journal of Economic Entomology, Lanham, v.78, p.681-686, 1985. Disponível em: <http://www.ingentaconnect.com/content/esa/ jee/1985/00000078/00000003/art00035\#expand/collapse>. Acesso em: 20 jun. 2007.

BOLLER, E.F. et al. Integrated production: principles and technical guidelines. 2.ed. Wädenswil: IOBC/WPRS/OILB/SROP,
1999. v.22, 29p. (Bulletin IOBC/WPRS, OILB/SROP, 4). Disponível em: <http://iobc-wprs.org/pub/index.html>. Acesso em: 13 nov. 2008 .

BOLLER, E.F. et al. Integrated production: principles and technical guidelines. 3.ed. Wädenswil: IOBC/WPRS/OILB/SROP, 2004. v.27, 54p. (Bulletin IOBC/WPRS, OILB/SROP, 2). Disponível em: <http://www.iobc.ch/iobc_bas.pdf>. Acesso em: 13 nov. 2008.

BOYERO, J.R. et al. Efectos de varios plaguicidas sobre Cryptolaemus montrouzieri Mulsant y Rhyzobius lophantae Blaisdell (Coleoptera: Coccinellidae). Boletín de Sanidad Vegetal de Plagas, Madri, v.31, n.1, p.79-87, 2005. Disponível em: <http://www.mapa.es/ministerio/pags/biblioteca/plagas/ BSVP-31-01-079-087.pdf>. Acesso em: 14 jun. 2006.

CLOYD, R.A.; DICKINSON, A. Effect of insecticides on mealybug destroyer (Coleoptera: Coccinellidae) and parasitoid Leptomastix dactylopii (Hymenoptera: Encyrtidae), natural enemies of citrus mealybug (Homoptera: Pseudococcidae). Journal of Economic Entomology, Lanham, v.99, n.5, p.1596-1604, 2006. Disponível em: <http://www.bioone.org/ doi/full/10.1603/0022-0493-99.5.1596>. Acesso em: 23 set. 2007. doi: 10.1603/0022-0493-99.5.1596.

CROFT, B.A. Arthropod biological control agents and pesticides. Environmental Science and Technology. New York: Wiley-Interscience, 1990. 723p.

DIB, G.A. Evaluación de la selectividad de pesticidas sobre Cryptolaemus montrouzieri Muls., enemigo natural de Pseudococcus spp. y Planococcus sp. 1998. 80f. (Tesis en Agronomia) - Universidad Catolica de Valparaiso. Facultad de Agronomia. Valparaiso, Chile.

FERNANDEZ, D.E. et al. Effects of seasonal mineral oil applications on the pest and natural enemy complexes of apple. Journal of Economic Entomology, Lanham, v.98, n.5, p.1630-1640, 2005. Disponível em: <http://www.bioone.org/ doi/full/10.1603/0022-0493-98.5.1630>. Acesso em: 23 set. 2007. doi: 10.1603/0022-0493-98.5.1630.

FERREIRA, D.F. Análises estatísticas por meio do Sisvar para Windows versão 4.0. In: REUNIÃO ANUAL DA REGIÃO BRASILEIRA DA SOCIEDADE INTERNACIONAL DE BIOMETRIA, 45., 2000, São Carlos. Anais... São Carlos, SP: UFSCar, 2000. p.255-258.

HASSAN, S.A.; DEGRANDE, P.E. Methods to test the side effects of pesticides on Trichogramma. In: PARRA, J.R.P.; ZUCHI, R.A. (Ed.). Curso de controle biológico com Trichogramma. Piracicaba, FEALQ, 1996. p.63-74.

HASSAN, S.A. Guidelines for testing the effects of pesticides on beneficial organisms: description of test methods. In: IOBC - INTERNATIONAL ORGANIZATION FOR BIOLOGICAL CONTROL. West Palaearctic Regional Section. Working Group "Pesticides and Beneficial Organisms". Abstracts... (IOBC/ WPRS) Bulletin, Southampton, v.15, p.1-186, 1992.

JANSEN, J.P.; HAUTIER, L. Comparative sensitivity of four ladybird species to five pesticides. In: VOGT, H. et al. IOBC/ WPRS - Working Group "Pesticides and Beneficial Organisms". Abstracts... (IOBC/WPRS) Bulletin, Poland: Meeting Debe, v.29, p.11, 2006. Disponível em: <http://www.iobc-wprs.org/ pub/bulletins/bulletin_2006_29_10_abstracts.pdf $>$. Acesso em: 18 ago. 2007. 
MAPA - Ministério da Agricultura, Pecuária e Abastecimento. Coordenação-Geral de Agrotóxicos e Afins/DFIA/DAS. Sistema Agrofit. 2010. Disponível em: <http://www.agricultura.gov.br>. Online. Acesso em: 23 dez. 2010.

MANZONI, C.G. et al. Seletividade de agrotóxicos recomendados na produção integrada da maçã a Trichogramma pretiosum Riley, 1879 (Hym.: Trichogrammatidae) em condições de laboratório. Revista Brasileira de Fruticultura, Jaboticabal, v.28, n.2, p.254-257, 2006. Disponível em: <http://www.scielo.br/pdf/rbf/ v28n2/a22v28n2.pdf>. Acesso em: 14 jan. 2007. doi: 10.1590/ S0100-29452006000200022.

MIRET, J.A.J.; GARCÍA-MARÍ, F. Side effects of pesticides on selected natural enemies occurring in citrus in Spain. IOBC/ WPRS Bulletin, Castelló de la Plana, v.24, n.4, p.103-112, 2001. Disponível em: <http://www.iobc-wprs.org/pub/bulletins/ iobc-wprs_bulletin_2001_24_04.pdf\#page=117>. Acesso em: 24 mar. 2005.

MORSE, J.G. et al. Residual toxicity of acaricides to three beneficial species on California citrus. Journal of Economic Entomology, Lanham, v.80, p.953-960, 1987. Disponível em: <http://www.ingentaconnect.com/content/esa/jee/1987/ 00000080/00000004/art00048>. Acesso em: 24 mar. 2005.

NARDO, E.A.B. et al. Perspectivas do controle biológico da praga quarentenária cochonilha-rosada no Brasil Maconellicoccus hirsutus (Green) (Hemiptera: Pseudococcidae). Jaguariúna: Embrapa Meio Ambiente, 1999. 38p. (Embrapa Meio Ambiente. Documentos, 2).

PMRA: Pest Management Regulatory Agency: PMRA List of Formulants, 2001.

RAIJ, B.V. Produção integrada de café - PIC. O Agronômico, Campinas, v.55, n.2, p.14-15, 2003. Disponível em: <http:// www.iac.sp.gov.br/OAgronomico/55-2/InfTecPIC.pdf $>$. Acesso em: 24 mar. 2005

RIPPER, W.E. et al. Selective insecticides and biological control. Journal of Economic Entomology, College Park, v.44, n.4, p.448-458, 1951.

ROCHA, L.C.D. et al. Seletividade fisiológica de inseticidas utilizados em cultura cafeeira sobre ovos e adultos de Cryptolaemus montrouzieri Mulsant. Arquivos do Instituto Biológico, São Paulo, v.77, n.1, p.119-127, 2010. Disponível em: <http:// www.biologico.sp.gov.br/docs/arq/v77_1/rocha.pdf >. Acesso em: 14 abr. 2010.
SANTA-CECÍLIA, L.V.C. et al. Cochonilhas-farinhentas em cafeeiros: bioecologia, danos e métodos de controle. Belo Horizonte: Epamig, 2007. 48p. (Boletim Técnico, 79).

SAMSØE-PETERSEN, L. Laboratory method for testing sideeffects of pesticides on the rove beetle Aleochara bilineata adults. IOBC/WPRS Bulletin, Southampton, v.15, n.3, p.8288, 1992. Disponível em: <http://www.springerlink.com/ content/h15128t261147576/>. Acesso em: 03 maio, 2007. doi: $10.1007 / \mathrm{BF} 02390933$.

SAS INSTITUTE. SAS/STAT: users guide: Introduction to Analysis of Variance Procedures. Cary, NC, 2001. Disponível em: <http://support.sas.com/documentation>. Acesso em: 23 maio, 2004.

SCOTT, A.J.; KNOTT, M.A. A cluster analyses method for grouping means in the analyses of variance. Biometrics, Raleigh, v.30, p.502-512, 1974. Disponível em: <http:// www.jstor.org/stable/2529204>. Acesso em: 08 maio, 2004.

TOMIZAWA, M.; CASIDA, J.E. Neonicotinoid insecticide toxicology: mechanisms of selective action. Annual Review of Pharmacology and Toxicology, Palo Alto, v.45, p.247-268, 2005. Disponível em: <http://www.annualreviews.org/doi/pdf/ 10.1146/annurev.pharmtox.45.120403.095930>. Acesso em: 28 nov. 2007. doi: 10.1146/annurev.pharmtox.45.120403.095930.

TOMIZAWA, M.; CASIDA, J.E. Selective toxicity of neonicotinoids attributable to specificity of insect and mammalian nicotinoid receptors. Annual Review of Entomology, Palo Alto, v.48, p.339-364, 2003. Disponível em: <http:// w w w. an nu a l reviews.org / d o i / a b s / 10.1146 / annurev.ento.48.091801.112731>. Acesso em: 08 nov. 2007. doi: 10.1146/annurev.ento.48.091801.112731.

VEIRE, M. van de. et al. Sequential testing scheme for the assessment of the side-effects of plant protection products on the predatory bug Orius laevigatus. BioControl, Dordrecht, v.47, n.1, p.101-113, 2002. Disponível em: <http:// www.springerlink.com/content/1bva63axwgmkt1ap/>. Acesso em: 03 mar. 2003. doi: 10.1023/A:1014473023912.

VOGT, H. Untersuchungen zu nebenwirkungen von insektiziden und akariziden auf Chrysoperla carnea Steph. (Neuroptera, Chrysopidae). Mededelingen Faculteit Landbouwkundige, Univ. Gent, v.57, n.2b, p.559-567, 1992.

WARE, G.W.; WhitACRE, D.M. An introduction to insecticides. 4.ed. Tucson, Arizona, 2004. Disponível em: $<$ http://ipmworld.umn.edu/chapters/ware.htm>. Online. Acesso em: 10 mar. 2009. 